\title{
Considering the Usability of End-User Security Software
}

\author{
Steven Furnell, Adila Jusoh, Dimitris Katsabas, and Paul Dowland \\ Network Research Group \\ University of Plymouth, Plymouth, United Kingdom \\ info@network-research-group.org
}

\begin{abstract}
Security features can now be found in a variety of end-user applications. However, the extent to which such features can actually be understood and used by the target audience is often undermined by poor attention to human-computer interaction factors. This paper considers the problem, and highlights a number of common issues that can compromise the usability of security features in practice. The discussion evidences the problems by means of examples from well-known applications, as well as drawing upon the results from a survey of over 340 end-users, which benchmarks the extent to which some of the observed issues actually affect them. It is concluded that users can currently face real difficulties, but could be relatively easily avoided though better design and implementation of the features concerned.
\end{abstract}

\section{Introduction}

In the face of an increasing volume and variety of threats, the use of security safeguards is an important consideration for end-user systems. However, getting users to realise and act upon this is often a challenge, and unless they have already had experience of a troublesome incident, users may need significant persuasion that security is something they need to worry about. Unfortunately, winning the awareness battle is only part of the challenge, and users can still be put off if they cannot understand and use the features that are placed before them. Sadly, this is often the reality of the situation, with security software that makes significant assumptions about users' technical capabilities and presents features in a manner that is more likely to reduce protection rather than encourage its use.

The importance of making systems usable is by no means a new discovery. Indeed, the principles of human-computer interaction ( $\mathrm{HCI}$ ) are now well-established, and a variety of information is now available to inform and guide the design of software to suit its intended user community [1,2]. In spite of this, it is still possible to identify many examples of poor practice, with the affected software being less easily used and understood as a result. The implementation of security features is an area that appears to fare particularly badly here, and this paper highlights a series of problems that can be commonly observed in relation to security functionality, based upon practical examples from a series of end-user applications.

Please use the following format when citing this chapter:

Author(s) [insert Last name, First-name initial(s)], 2006, in IFIP International Federation for Information

Processing, Volume 201, Security and Privacy in Dynamic Environments, eds. Fischer-Hubner, S., Rannenberg, K., Yngstrom, L., Lindskog, S., (Boston: Springer), pp. [insert page numbers]. 


\section{Common Problems in End-User Security Features}

This section highlights a number of examples of common failings that are apparent in programs that target end-users. For the purposes of discussion, five key themes are used to group the problem issues. However, it should be noted that several of the points are inter-related, and the practical examples used to illustrate them can certainly be seen to be suffering from more than one of the problems. It should also be noted that none of these issues are specifically related to the implementation of security functionality, and indeed the themes identified here are closely related to usability heuristics proposed by Nielsen for systems in general [3].

In addition to practical examples, the discussion draws upon the results from an online survey of end-users, which was conducted in order to assess understanding of application-level security features amongst the general user population. The survey presented respondents with screenshots relating to the security functionality within a number of popular end-user applications, and attempted to determine whether they were meaningful (e.g. in relation to the terminology used) and correctly interpreted (i.e. whether the intention of the functionality was properly understood). A total of 342 responses were received, and the main characteristics of the respondent group were as follows:

- almost equally split between male and female

- over $80 \%$ in the $17-29$ age group

- over $80 \%$ have university-level education

- over $96 \%$ are regularly use a computer at home and/or at work

- almost $90 \%$ rated themselves as intermediate or advanced users

These factors suggest that the respondents as a whole were likely to have a high level of IT literacy, making them well-placed to provide relevant comments about the usability of security features within the targeted applications. Some of the significant findings from the survey are therefore used to support the discussion presented here. For readers interested obtaining further information, the full details of the survey and the associated results can be found in [4].

\subsection{Reliance upon Technical Terminology}

One of the traditional barriers to newcomers into IT is the significant degree of technical terminology that accompanies the domain. Over time, efforts have been made to ease this burden, with increased use of pictures and plain language as a means of expressing concepts to novices. However, security is one area in which the message is still very likely to be unclear, with technical terms often being an intrinsic part of how features are conveyed. An example of this problem is illustrated in Figure 1, which shows the means by which users are able to set the 'security level' for browsing sites within Internet Explorer (IE). Provided as the standard browser within the most popular operating system environments, IE is, of course, the means by which most users come into contact with the Web, and browsing is a context in which appropriate security is most definitely required. However, although the interface initially looks quite straightforward, with the use of a slider control to set the desired security level on a four-point scale (low, medium-low, medium, and high), it becomes 
somewhat less intuitive if users try to understand the descriptions of the settings. For example, one of the characteristics of the 'medium' setting described in the Figure is that "Unsigned ActiveX controls will not be downloaded". Although this would be unlikely to cause problems for users with a technology background, it has clear potential to confuse the average user (who might nonetheless have an interest in setting up their system securely, and so could certainly find themselves looking at the related options). As a result, while they will appreciate the idea of the low-to-high scale, the descriptions may impede their ability to relate this to their browsing needs.

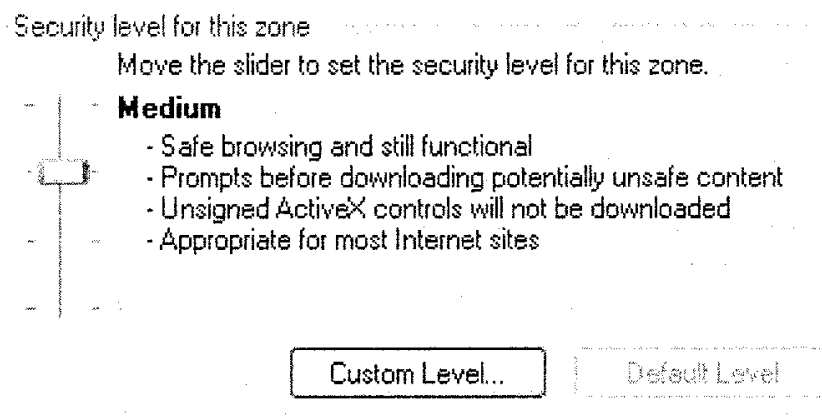

Fig. 1. Setting the 'Security level' within Internet Explorer.

Respondents to the authors' survey were presented with this screenshot, and asked to indicate whether they understood it. The results revealed that $34 \%$ did not. Although this is already a sizeable proportion of users to lose, the authors anticipated that some respondents would claim to understand the interface even though they did not actually understand all of the terminology. As such, the questionnaire proceeded to ask whether respondents had heard of ActiveX before, and if so, whether they actually knew what it meant. Although the initial finding here was mostly positive, with $65 \%$ claiming to have heard of the term, only $54 \%$ of these people (i.e. only $35 \%$ of the overall respondent group) knew the meaning. This puts a rather different interpretation upon the proportion of people who would fully understand the setting in Figure 1, with almost two thirds of the overall respondent group unable to comprehend the complete description.

\subsection{Unclear and Confusing Functionality}

If users are confronted with security features that they do not understand, then it increases the chance that they will make mistakes. In some cases, these mistakes will put their system or data at increased risk, whereas in others they may serve to impede the user's own use of the system. Confusion can often arise from the way in which features are presented, with the result that even the most straightforward and familiar security safeguards can become challenging to use. As an example, we can consider the way in which password protection is used in several Microsoft Office applications. Excel, PowerPoint and Word all allow two levels of password to be 
applied to user files - to control access to the file (i.e. in order to maintain confidentiality), or to restrict the ability to modify it (i.e. controlling integrity). Such passwords are set via two distinct fields in the 'Security' tab that users can access from the Tools-Options menu (as depicted in the left side of Figure 2). Users who subsequently attempt to open such a password-protected file are then presented with the dialogs on the right-hand side of Figure 2 (the prompts in this case being taken from Microsoft Word). However, whereas the prompt for opening an accessedcontrolled file (the upper dialog) is relatively easy to understand (i.e. you need a password and cannot open the document without it), the dialog for files that are merely protected against modification is often misunderstood. Users who wish to simply view or print the file can select the 'Read Only' option, in order to bypass the password request. However, the presentation of the interface causes confusion in practice, and many users are so distracted by the apparent requirement for a password that they believe they cannot do anything without it.

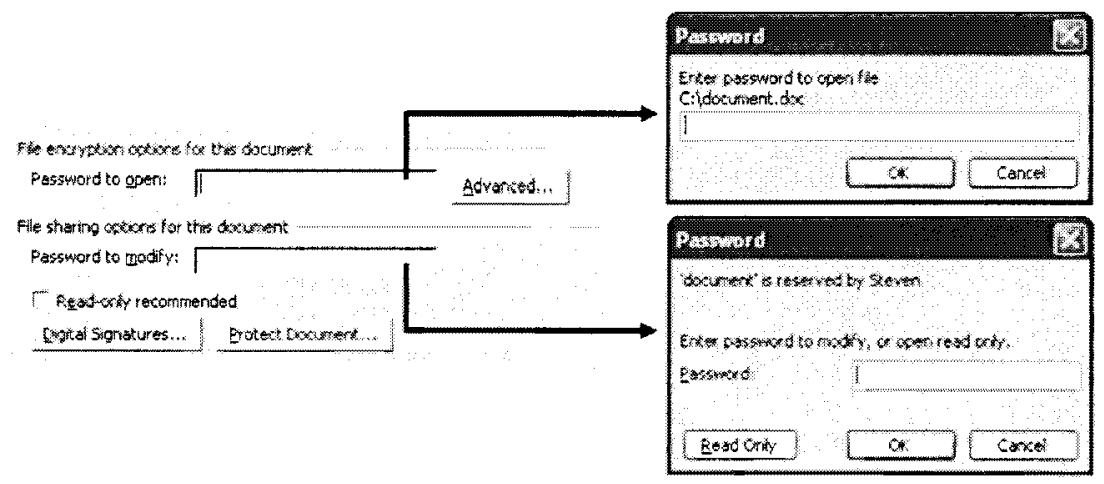

Fig. 2. Password options and the resulting prompts within Microsoft Word.

In the authors' survey, respondents were presented with an example of the lower password dialog, and asked to indicate which of three options they understood it to mean. Although the majority correctly indicated that it meant the document could not be modified without a password, $23 \%$ incorrectly believed that the file could not be opened without a password, and a further $13 \%$ were not sure how to interpret it. As such, more than a third of users would not have been in a position to make the correct decision.

\subsection{Lack of Visible Status and Informative Feedback}

Users ought to know when security is being applied and what level of protection they are being given. This not only provides a basis for increasing their confidence when using particular services, but can also remind them to configure the system correctly. Without such a reminder, users may proceed to perform sensitive tasks without adequate protection, or may inadvertently leave settings at a level that impedes their legitimate usage. As such, the lack of visible status information is another example of 
undesirable HCl. As an illustration of how this can cause problems, the screenshots in Figure 3 show two attempts to reach Microsoft's Hotmail service via Internet Explorer, with the browser security level set to 'high'.

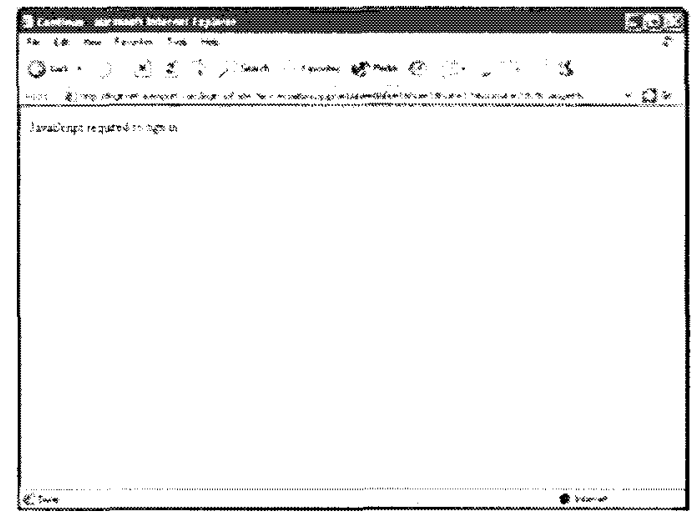

(a)

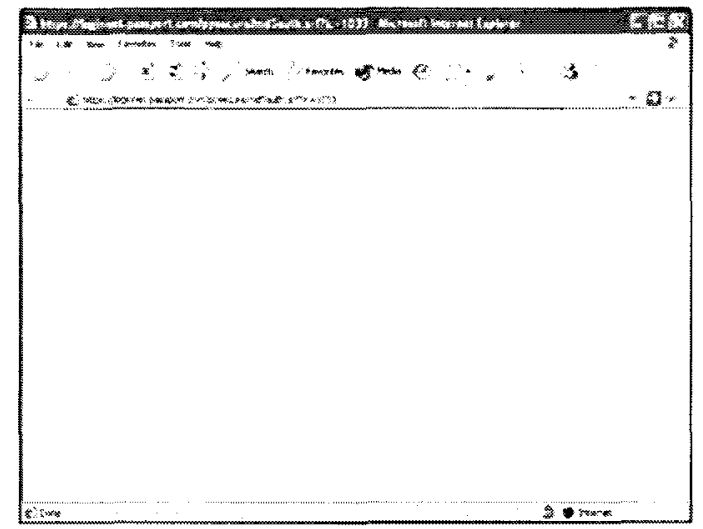

(b)

Fig. 3. Attempts to access Hotmail with Internet Explorer security set to 'high' - (a) directly via URL (b) indirectly via MSN Messenger.

Figure 3a shows the effects of attempting to reach the service by typing the 'www.hotmail.com' into the address bar. The result is a message advising "JavaScript required to sign in" - which would be unlikely to be a sufficiently informative message to alert many users to the fact that their security settings were preventing the site from loading correctly. Meanwhile, Figure $3 \mathrm{~b}$ shows an even worse scenario, with the user having attempted to log in via the MSN Messenger service. In this case, the user receives no message at all, and there is no indication of what the problem might be. As such, they may conclude that the site is simply not operational. What the user should receive is a clear message to remind them that their 
browser security is set to 'high', and to indicate that this may cause the site to operate incorrectly.

\subsection{Forcing Uninformed Decisions}

Even if users do not go looking for security-related options and attempt to change the settings, they may still find themselves confronted with the need to take securityrelated decisions during the course of their normal activities. In these contexts, it should be all the more important for the information to be conveyed to them in a meaningful fashion, with minimal assumptions of prior knowledge and maximum help available to ease the process. Unfortunately, however, users may again find themselves at a disadvantage in practice, with dialogs often being conveyed in a manner that only advanced participants would be comfortable with. To illustrate the point, Figures 4 and 5 present two examples of dialogs that may be encountered by users during standard web browsing activities. The first example illustrates the type of warning that a user would receive when a website's security certificate has been issued by a provider that is not specified as trusted in their security configuration. This does not, of course, mean that the certifying authority cannot be trusted, but the user is being asked to check in order to make a decision. The likely problem here is that most users will not know what a security certificate is, let alone be able to make a meaningful decision about one. Meanwhile, the example in Figure 5 is warning the user that a web page they are attempting to download contains active content that could potentially be harmful to their system. The difficultly for most users is again likely to be that they would not understand what they were being asked, with terms such as 'active content' and 'ActiveX' being more likely to confuse than explain. Of course, part of the problem in these examples relates to the earlier issue of using technical terminology. However, the problem here goes somewhat deeper, in the sense that both cases are obliging the user to make a decision without the option to seek further help from the system. As such, they would be forced to make a decision in the absence of sufficient information. As an indication of the scale of this problem, the example from Figure 5 was presented to the survey respondents, and $56 \%$ indicated that they would not know how to make a decision.

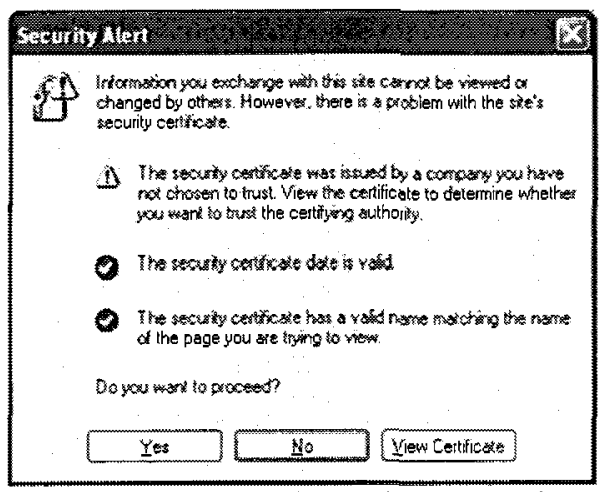

Fig. 4. Website security certificate warning. 


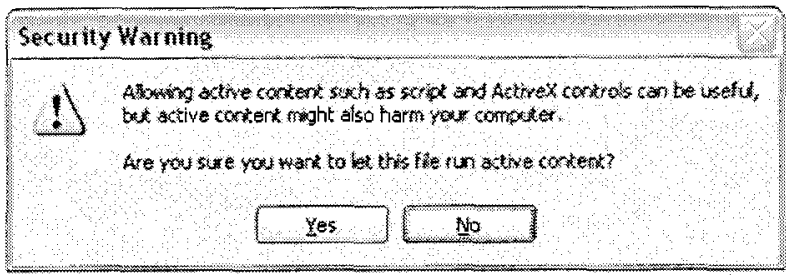

Fig. 5. Active content warning.

\subsection{Lack of Integration}

Another way in which the presentation of security features may serve to confuse users is if different aspects do not integrate together in a meaningful fashion. Although individual mechanisms are often provided in different software from different vendors, it would not be unreasonable for users to expect security features to work in concert. Unfortunately, it is possible to identify examples in which such integration does not occur, and users receive contradictory messages as a consequence. As an example, Figure 6 a shows the security settings for macro functions within Microsoft Word. The significant part of the image is near the very bottom, with the indication that no virus scanner is installed. In actual fact, this screenshot was taken from a machine running McAfee VirusScan Enterprise 7 (the icon for which is visible as the third item in the system tray), and so receiving a message claiming that no virus protection is installed is hardly useful to the user. Meanwhile, Figure $6 \mathrm{~b}$ presents an example of a pop-up message that appeared on a Dell PC during normal daily usage. Although this could be considered useful as a friendly reminder to general users that they need to be concerned about security, the problem in this case was that the message popped up on a machine running Norton Internet Security - which meant it already had the firewall and anti-virus protection being referred to. Some users will interpret the wording of the message ("you should have a firewall...") to mean that the system is telling them that they are not adequately protected - which could cause obvious confusion and concern for users who considered they already had suitable safeguards. It would be preferable to offer more specific advice, tailored to user's actual circumstances (e.g. "You have a firewall and virus protection, but should also have anti-spyware protection installed"). Failing this (e.g. if it was not possible to determine existing protection status), the wording could still be adjusted to something that would pose a question rather than make an apparent statement (e.g. "Do you have a firewall?"), and therefore allow users who knew they were protected to pass by without concern. 


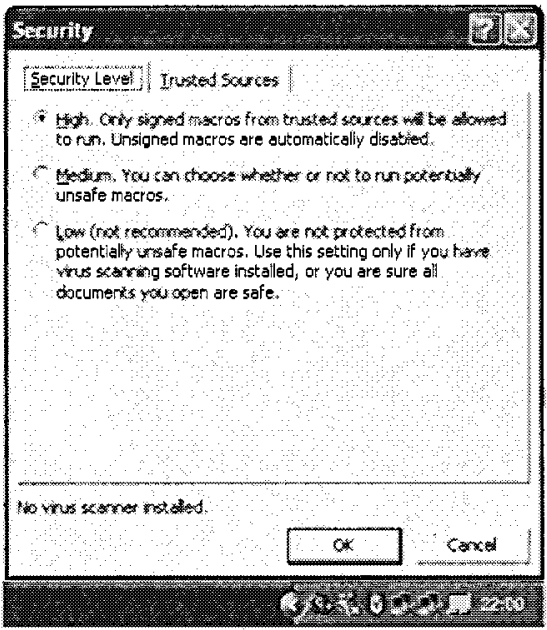

(a)

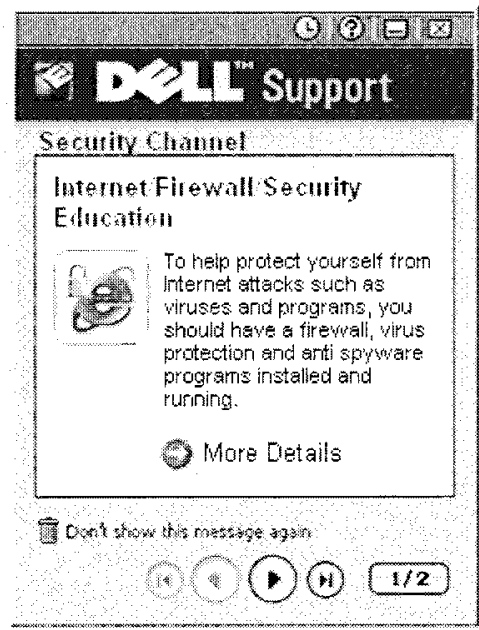

(b)

Fig. 6. Examples of misinformation due to lack of integration.

Although the messages in both of these examples aimed to be helpful, warning and reminding users that security needs to be considered, it could be argued that if the system is unable to give an accurate message that it would be preferable to say nothing at all.

\section{Improving the Situation}

Appropriate presentation of security features is unlikely to happen by chance, and software designers should therefore pay specific attention to making sure that their systems are easily understood and used by the target audience. Of course, attempting to reach such a goal ought not to be an ad hoc endeavour, and the aforementioned good practice in domains such as HCI may be used to assist the process. In addition, a number of published works specifically consider guidelines for developing usable security systems $[5,6]$.

Having identified a series of less desirable examples, it is also relevant to observe that many examples of stronger interface design can be found. As an illustration, Figure 7 presents two screenshots taken from the Norton Internet Security package, which aims to provide an integrated security solution (including firewall, anti-virus, anti-spam, and intrusion detection) for end-user systems. The tool, of course, differs from the earlier examples in the paper, because it represents an example of software that has been specifically designed to fulfil a security role, rather than a wider application within which security is just one of the supporting functions. As such, it can be assumed that the designers and developers would have been in a position to devote more specific attention to the presentation and usability of the protection 
features. As a result, some of the positive observations arising from this particular interface are that:

- all of the top-level security options are visible and configurable from a single window;

- the status of each option is clearly conveyed, along with the consequent security status of the overall tool;

- brief and clearly-worded explanations are provided to accompany each main option, and further help is easily accessible in each case.
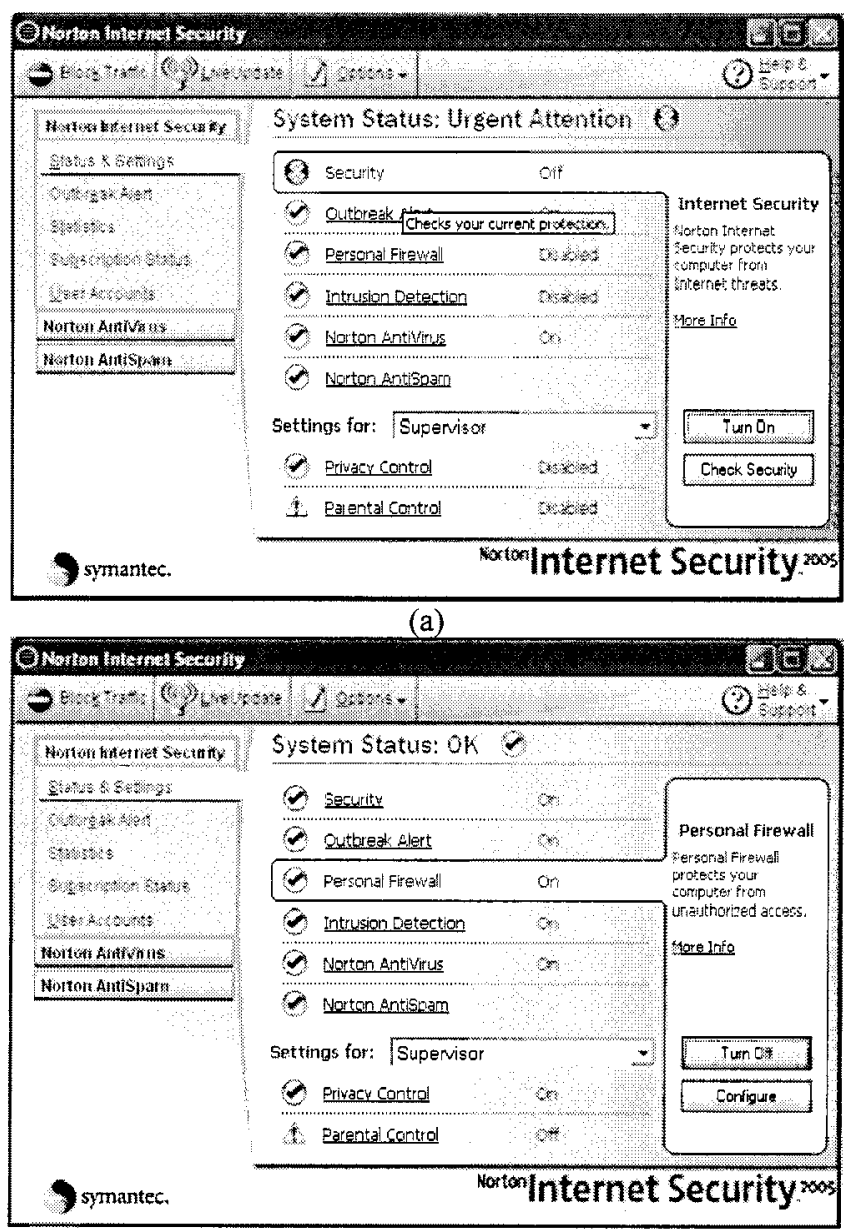

(b)

Fig. 7. Examples of stronger interface design

In Figure 7a, the user receives a clear visual indication that things are amiss, with the 'Urgent Attention' banner and the warning icon beside the aspect that is causing 
the concern (in addition, the background of the information area is shaded red). Meanwhile, the next dialogue (Figure $7 \mathrm{~b}$ ) shows the system to be in the more desirable state of having all the critical elements working correctly (note that although the 'Parental Control' option is disabled, it does not affect the overall security status, and the window is now shaded with a reassuring green background). Comparing the dialogs to the earlier examples, it is apparent that none of the previous problems are immediately on show.

\section{Conclusions}

If users are to be expected to make use of security, then it is clearly important for the related features to be presented in a manner that they can understand and use. Unfortunately, appropriate attention to these issues is far from guaranteed, and the paper has highlighted a number of problems that can be encountered in current software. However, it is notable that all of the problems indicated here would essentially have been avoidable by taking a more considered approach to the design of the user interfaces. Future developments should therefore seek to avoid repeating the same problems. In support of this, research should aim to better understand the ways in which end-users relate to security, in order to help realise interfaces and interaction styles suited to their varying backgrounds and abilities. The authors are currently pursuing research in this area, accompanied by hands-on trials in order to enable a deeper insight into how users understand and interact with the security features already at their disposal. The results from these activities will help to inform the design of alternative approaches, which will also be evaluated and reported as part of future work.

\section{References}

1. Shneiderman, B. 1998. Designing the User Interface: Strategies for Effective HumanComputer Interaction. (3rd ed.). Menlo Park, CA: Addison Wesley.

2. Carroll, J.M. 2001. Human-Computer Interaction in the New Millennium, Addison-Wesley Professional.

3. Nielsen, J. 1994. "Heuristic evaluation", in Nielsen, J. and Mack, R.L. (Eds.), Usability Inspection Methods, John Wiley \& Sons, New York.

4. Furnell, S.M., Jusoh, A. and Katsabas, D. 2006. "The challenges of understanding and using security: A survey of end-users", Computers \& Security, vol. 25, no.1.

5. Johnston, J., Eloff, J.H.P. and Labuschagne, L. 2003. "Security and human computer interfaces", Computers \& Security, vol. 22, no. 8, 675-684.

6. Katsabas, D., Furnell, S.M., Dowland, P.S. 2005. Using Human Computer Interaction principles to promote usable security. Proceedings of the Fifth International Network Conference (INC 2005), Samos, Greece, 235-242. 